\title{
EDITORIAL
}

\section{Closing the GAPP on pneumonia}

\author{
Pneumonia kills more children under 5 years of age than any other infectious disease, but \\ affordable and effective treatment and prevention measures are available. The time has \\ come for the world to wake up to this forgotten killer.
}

Which disease is the biggest killer of children under 5 years of age worldwide? When asked this question, it is likely that most microbiologists would think of highprofile diseases such as HIV/AIDS, malaria and tuberculosis. Between them, the 'big three' account for more than 1 million deaths in children under 5 years of age each year and, in 2007, attracted US\$1.96 billion in research and development investment ${ }^{1}$. But in fact, the biggest killer of children under 5 years of age is pneumonia. This disease kills an estimated 2 million children in this age group each year, more than HIV/AIDS, measles and malaria combined. However, in 2007 pneumonia and meningitis received only $\$ 33$ million in research and development investment ${ }^{1}$. It is little wonder that pneumonia is sometimes referred to as the forgotten killer.

Pneumonia can be caused by a range of bacterial, viral and fungal respiratory pathogens, either alone or in combination. The most common causes of the disease are Streptococcus pneumoniae (the pneumococcus) and Haemophilus influenzae type b (Hib), both of which are also leading causes of meningitis. Global estimates of the burden of disease caused by these bacterial pathogens in 2000 were recently published ${ }^{2,3}$. Together, they accounted for $\sim 1.2$ million deaths in children aged 1-59 months; Hib infection caused an estimated 371,000 deaths and 8.13 million cases of serious illness, and the pneumococcus caused an estimated 826,000 deaths and 14.5 million cases of serious illness.

Pneumonia accounts for one-fifth of all deaths of children under 5 years of age worldwide, $98 \%$ of which occur in just 68 developing countries. The tragedy is that pneumonia is preventable and treatable. Pneumonia can be treated effectively with antibiotics, but they are prescribed to less than $20 \%$ of affected children. Several vaccines that can prevent pneumonia have been available for many years, some for decades. Two of these, the measles and pertussis vaccines, have been incorporated into routine vaccination programmes in many countries, where they have been successful in reducing pneumonia morbidity and mortality. However, for the newer vaccines that tackle the two most common causes of pneumonia, the Hib vaccine and the pneumococcal conjugate vaccine (PCV), uptake has been far less widespread, particularly for PCV.
Why has progress in tackling this preventable and treatable disease lagged behind progress in tackling other diseases? One reason could be that, in terms of media coverage, political and social awareness, and funding, pneumonia has been severely neglected compared with higher-profile diseases such as HIV/AIDS and malaria. In an attempt to redress the balance, on 2 November 2009, the first World Pneumonia Day, a multi-agency consortium launched the Global Action Plan for the Prevention and Control of Pneumonia (GAPP). GAPP aims to raise the profile of pneumonia as a public-health emergency and spur the relevant stakeholders into action. The strategy is based on three main principles: protect, through measures such as encouraging and supporting breastfeeding, reducing the rates of low birth weight and improving nutrition; prevent, by expanding and implementing vaccination programmes and controlling indoor pollution; and treat, by supporting effective case management and diagnostics. Many of the resources needed to tackle pneumonia are already available, and the aim of the GAPP taskforce is to galvanize local, national and international authorities into dedicating the necessary resources to combating the problem. In terms of the immunization programmes, help is available from the GAVI Alliance, which provides financial support for immunization to 72 low-income countries that represent more than half of the world's population. To date, the GAVI Hib initiative has supported the introduction of the Hib vaccine in 59 of the 72 eligible countries, and a major push to expand access to PCV to an additional 130 million children by 2015 has been announced.

One child dies from pneumonia every 15 seconds. Tackling pneumonia effectively will greatly assist progress towards achieving the fourth United Nations Millennium Development Goal: to reduce childhood mortality by one-third from the 1990 baseline by 2015 . We have the knowledge to be able to substantially reduce morbidity and mortality from pneumonia. It is time to put this knowledge into action.

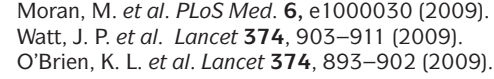

